\title{
Dynamic Changes in Portal Vein Flow during Decongestion in Patients with Heart Failure and Cardio-Renal Syndrome: A POCUS Case Series
}

\author{
Eduardo R. Argaiz ${ }^{a}$ Philippe Rolab Gerardo Gamba ${ }^{a, c}$ \\ aDepartment of Internal Medicine, Instituto Nacional de Ciencias Médicas y Nutrición Salvador Zubirán, Tlalpan,

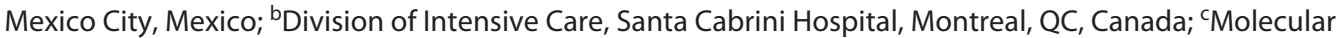 \\ Physiology Unit, Instituto de Investigaciones Biomédicas, Universidad Nacional Autónoma de México, Mexico City, \\ Mexico
}

\section{Keywords}

Point-of-care ultrasound - Portal vein pulsatility index .

Venous congestion · Cardio-renal syndrome

\begin{abstract}
Introduction: Optimal method for noninvasive assessment of venous congestion remains an unresolved issue. Portal vein (PV) and intrarenal venous flow alterations are markers of abdominal venous congestion and have been associated with acute kidney injury (AKI) in cardiac surgery patients. It is currently unknown if portal vein flow (PVF) alterations in heart failure can be reversed with diuretic treatment and track decongestion. Objective: The aim of this study is to evaluate PVF alterations in patients with ADHF at arrival and after decongestive treatment. Methods: Assessment of venous congestion using point-of-care ultrasound was performed in 12 patients with ADHF ( 6 patients with left-sided heart failure and 6 patients with right-sided heart failure). Evaluation included inferior vena cava (IVC) size and collapsibility in addition to PV Doppler to determine pulsatility fraction (PF). Results: Increased PV PF (81.75 $\pm 13 \%)$ was found on admission. After effective decongestive treatment, it improved to $(17.43 \pm 2.2 \%)$. Improvement in IVC size and collapsibility was seen in most patients with left-sided heart fail-
\end{abstract}

ure and none of the patients with right-sided heart failure. Improvement in PV PF coincided with return to baseline of Serum $\mathrm{Cr}$ in patients that presented with AKI. Conclusions: Evaluation of abdominal venous congestion by point-ofcare ultrasound could aid in diagnosis and follow-up of patients with congestive kidney injury.

(c) 2021 S. Karger AG, Basel

\section{Introduction}

Hospitalizations from heart failure have been shown to be preceded by a gradual increase in cardiac filling pressures using invasive ambulatory monitoring measurements [1,2]. Noninvasive methods for assessment of congestion are still being developed. While bioelectrical vectorial impedance analysis has been proposed as an aid to evaluate body hydration status [3], this technique does not directly assess congestion. The use of point-of-care ultrasound (POCUS) to evaluate lung congestion has been shown to decrease decompensations and urgent heart failure visits $[4,5]$. However, besides lung congestion, the abdominal compartment contributes significantly to deranged cardiac as well as renal function in congestive heart failure (CHF) [6]. karger@karger.com

(c) 2021 S. Karger AG, Basel

www.karger.com/crm

Karger ${ }^{\prime}=$
Eduardo R. Argaiz

Department of Internal Medicine

Instituto Nacional de Ciencias Médicas y Nutrición Salvador Zubiran

Vasco de Quiroga No. 15, Tlalpan, Mexico City 14080 (Mexico)

lalo.argaiz@gmail.com 
Table 1. Demographic and clinical characteristics

\begin{tabular}{|c|c|c|c|}
\hline & $\begin{array}{l}\text { All patients, } \\
n=12\end{array}$ & $\begin{array}{l}\text { Left-sided } \\
\text { heart disease, } \\
n=6\end{array}$ & $\begin{array}{l}\text { Right-sided } \\
\text { heart disease, } \\
n=6\end{array}$ \\
\hline \multicolumn{4}{|l|}{ Demographics } \\
\hline Age, years & $56 \pm 15$ & $66 \pm 9$ & $46 \pm 12$ \\
\hline Male, \% & 67 & 83 & 50 \\
\hline \multicolumn{4}{|l|}{ Medical history, \% } \\
\hline Diabetes & 67 & 83 & 50 \\
\hline Hypertension & 67 & 100 & 33 \\
\hline CKD & 67 & 100 & 33 \\
\hline Ischemic heart disease & 25 & 50 & 0 \\
\hline \multicolumn{4}{|l|}{ NYHA class, $\%$} \\
\hline I & 0 & 0 & 0 \\
\hline II & 41 & 33 & 50 \\
\hline III & 33 & 33 & 33 \\
\hline IV & 25 & 33 & 16 \\
\hline \multicolumn{4}{|l|}{ Medications, \% } \\
\hline$\beta$-Blocker & 50 & 83 & 16 \\
\hline ACE inhibitor or ARB & 33 & 67 & 0 \\
\hline Loop diuretic & 58 & 83 & 33 \\
\hline Spironolactone & 25 & 33 & 16 \\
\hline \multicolumn{4}{|l|}{ Admission laboratory findings } \\
\hline Serum sodium, $\mathrm{mEq} / \mathrm{L}$ & $134 \pm 3$ & $134 \pm 3$ & $135 \pm 3$ \\
\hline Hemoglobin, g/dL & $11.7 \pm 3$ & $10.7 \pm 1.6$ & $12.6 \pm 4.8$ \\
\hline B-type natriuretic peptide, $\mathrm{pg} / \mathrm{mL}$ & $2,085 \pm 2,359$ & $2,830 \pm 2,920$ & $1,190 \pm 1,193$ \\
\hline Glomerular filtration rate, $\mathrm{mL} / \mathrm{min}$ & $32 \pm 29$ & $17 \pm 7$ & $46 \pm 37$ \\
\hline \multicolumn{4}{|l|}{ Echocardiographic parameters } \\
\hline LVEF, \% & $55 \pm 17$ & $42 \pm 14$ & $68 \pm 5$ \\
\hline $\mathrm{E} / \mathrm{e}^{\prime}$ & $16 \pm 8$ & $21 \pm 8$ & $12 \pm 6$ \\
\hline TAPSE, mm & $17 \pm 3$ & $17 \pm 3$ & $18 \pm 4$ \\
\hline S' wave & $11 \pm 4$ & $11 \pm 3$ & $12 \pm 6$ \\
\hline RV fractional area change, $\%$ & $38 \pm 13$ & $43 \pm 12$ & $32 \pm 14$ \\
\hline Max tricuspid regurgitation velocity, $\mathrm{cm}$ & $3.4 \pm 0.9$ & $3.2 \pm 0.5$ & $3.7 \pm 1.2$ \\
\hline
\end{tabular}

There is increasing recognition that worsening renal function in CHF is related to altered renal blood flow $[7,8]$. Recently, alterations in renal venous flow (IRVF) assessed by Doppler imaging have been associated with worse outcomes in patients with CHF $[9,10]$. Besides IRVF alterations, portal vein flow (PVF) alterations have been proposed as a marker of venous congestion and right ventricular dysfunction $[11,12]$. PVF and IRVF alterations have been shown to correlate with each other and were independently associated with the development of subsequent acute kidney injury (AKI) in patients undergoing cardiac surgery $[13,14]$. Portal vein (PV) Doppler is easily obtainable and less time consuming than intrarenal venous Doppler [13] Given the potential usefulness of evaluating venous congestion via POCUS of PVF, we decided to study the dynamic changes that occur during decongestion in patients presenting with heart failure to the emergency department.

\section{Materials and Methods}

\section{Study Population}

Patients were included prospectively from July 2019 to January 2020. Enrollment occurred following an advanced POCUS consult from the attending emergency department physician. The advanced POCUS consult is mainly focused on evaluating and grading the severity of venous congestion by analyzing venous Doppler waveforms. Given that POCUS evaluation of venous congestion was performed only in patients whose emergency department physician solicited consultation, this resulted in a convenience sample.

Eligible patients were patients aged 18 or over, presenting to the emergency department of a single tertiary care center with the diagnosis of acute decompensated heart failure (either de novo HF or chronic decompensated HF) fulfilling the following criteria: new or worsening HF symptoms (including dyspnea, decreased exercise capacity, fatigue, or other volume overload symptoms), physical examination findings compatible with $\mathrm{HF}$ (peripheral edema, pulmonary congestion, increased jugular venous pressure or third heart sound), and point-of-care ultrasound (POCUS) ex-
60

Cardiorenal Med 2021;11:59-66 DOI: $10.1159 / 000511714$
Argaiz/Rola/Gamba 
amination showing severe abdominal venous congestion (severe abdominal venous congestion was defined as a plethoric inferior vena cava (IVC) [IVC diameter $>2 \mathrm{~cm}$ with $<20 \%$ inspiratory collapse] plus a PV pulsatility fraction (PF)

$$
\left(\frac{V_{\max }-V_{\min }}{V_{\max }} \times 100\right)
$$

$>30 \%$ as previously reported [13]). Exclusion criteria included lack of will to participate, cardiogenic shock, or hemodynamic instability. Patients with previously diagnosed Child-Pugh B or C liver cirrhosis, liver transplant, or ESRD on renal replacement therapy were also excluded. Medical history to determine the presence of these comorbidities was obtained from electronic medical record. We also excluded patients with intrinsic AKI as determined by consulting nephrologist with the aid of urinalysis, urine sediment, and time course of reversibility.

\section{Study Protocol}

All patients had an initial POCUS examination on arrival consisting of IVC size and collapsibility index and PV Doppler. Hepatic vein Doppler was obtained to assure pulsatile PVF reflected changes in right atrial pressure. Examination was repeated after decongestive treatment in all patients. Severe abdominal venous congestion was defined as a plethoric IVC (IVC diameter $>2 \mathrm{~cm}$ with $<20 \%$ inspiratory collapse) plus a PV PF

$$
\left(\frac{V_{\max }-V_{\min }}{V_{\max }} \times 100\right)
$$

$>30 \%$ [13]. The IVC diameter was measured in its intrahepatic portion at $2 \mathrm{~cm}$ of the junction with the hepatic veins using a longitudinal view from a subxiphoid position. The complete method for hepatic and portal Doppler assessment has been previously published [13]. POCUS was performed with a Sonosite Edge device, and a phased array probe using the predefined abdominal settings. All measurements were repeated 3 times and only considered valid if variability between measurements was $<10 \%$. Diuretic treatment was selected at the discretion of treating clinicians with input from POCUS findings. Successful decongestion was considered in patients with clinical improvement in symptoms and signs of congestion and/or lower brain natriuretic peptide. The Institutional Review Board approved the study with waiver of patient consent (NMM-3393). Statistical analysis was performed on Prism 8 (GraphPad Software, San Diego, CA, USA) using paired Student's $t$ test.

\section{Results}

A total of 12 patients were included (Table 1). Six patients had left-sided heart failure (heart failure with reduced ejection fraction and heart failure with preserved ejection fraction) (patients 1-6), while 6 had right-sided heart failure (pulmonary arterial hypertension and constrictive pericarditis who were not surgical candidates) (patients 7-12). At the time of diagnosis, all patients had a plethoric IVC and a PV PF $>30 \%$. After diuretic treatment, all patients were able to be decongested as evidenced by clinical improvement, negative cumulative fluid balance (mean $\pm \mathrm{SD})(-8.2 \pm 4 \mathrm{~L})$, and a decrease in brain natriuretic peptide $(2,085 \pm 2,359 \mathrm{pg} / \mathrm{mL}$ before decongestion vs. $606 \pm 474 \mathrm{pg} / \mathrm{mL}$ after decongestion $p=$ 0.032). Three patients had $>2$ POCUS evaluations and showed progressive improvements in portal flow alterations with decongestion (Fig. 1). All patients showed improvement in PV PF (81.75 $\pm 46 \%$ before decongestion vs. $17.43 \pm 8 \%$ after decongestion $p=0.0003$ ) (Fig. $2 \mathrm{a}$ ). While most patients with left-sided heart failure had a nonplethoric IVC after treatment (66.6\%), this was not achieved in any of the patients with right-sided heart failure (Fig. 2b). A total of 8 patients (patients 3-10) presented with congestive AKI (Serum Cr [SCr] $3.42 \pm 1.94$ $\mathrm{mg} / \mathrm{dL}$ at presentation), and it resolved in all of them after decongestion (SCr $1.87 \pm 0.92 \mathrm{mg} / \mathrm{dL}$ ). Only 6 patients had available urinalysis. None showed increased proteinuria or hematuria. Urine sediment was bland in all cases. Urinary sodium was $<20 \mathrm{mEq} / \mathrm{L}$ in 4 patients, and $>30 \mathrm{mEq} / \mathrm{L}$ in 2 patients with recent administration of IV loop diuretic. No other cause of AKI was identified (Table 2).

\section{Discussion}

In this study, we present a case series of patients with acute decompensated heart failure and point-of-care ultrasound evidence of abdominal venous congestion evidenced by a mean PV PF of $81.75 \%$. We saw a high incidence of congestive AKI (67\%) likely secondary to selection: patients with $\mathrm{ADHF}$ and $\mathrm{AKI}$ might be more likely to trigger an advanced POCUS consult from ED physicians than patients with $\mathrm{ADHF}$ without $\mathrm{AKI}$. The fact that only patients with severe venous congestion were included could have also played a role. Given exclusion of patients with intrinsic AKI, all patients were able to be decongested with diuretic treatment. Decongestion was accompanied by an improvement in PV PF in all patients. A nonplethoric IVC was achieved in most patients with left-sided heart failure but not in those with right-sided heart failure. While previous studies have suggested that a false positive increase in PV PF can be present in healthy individuals with low BMI [15], we aimed to increase the specificity of this exam by adding the evaluation of the IVC to the study protocol. In fact, a previous study has shown that multiple-site evaluation of venous congestion including IVC, hepatic, portal, and intrarenal veins in- 


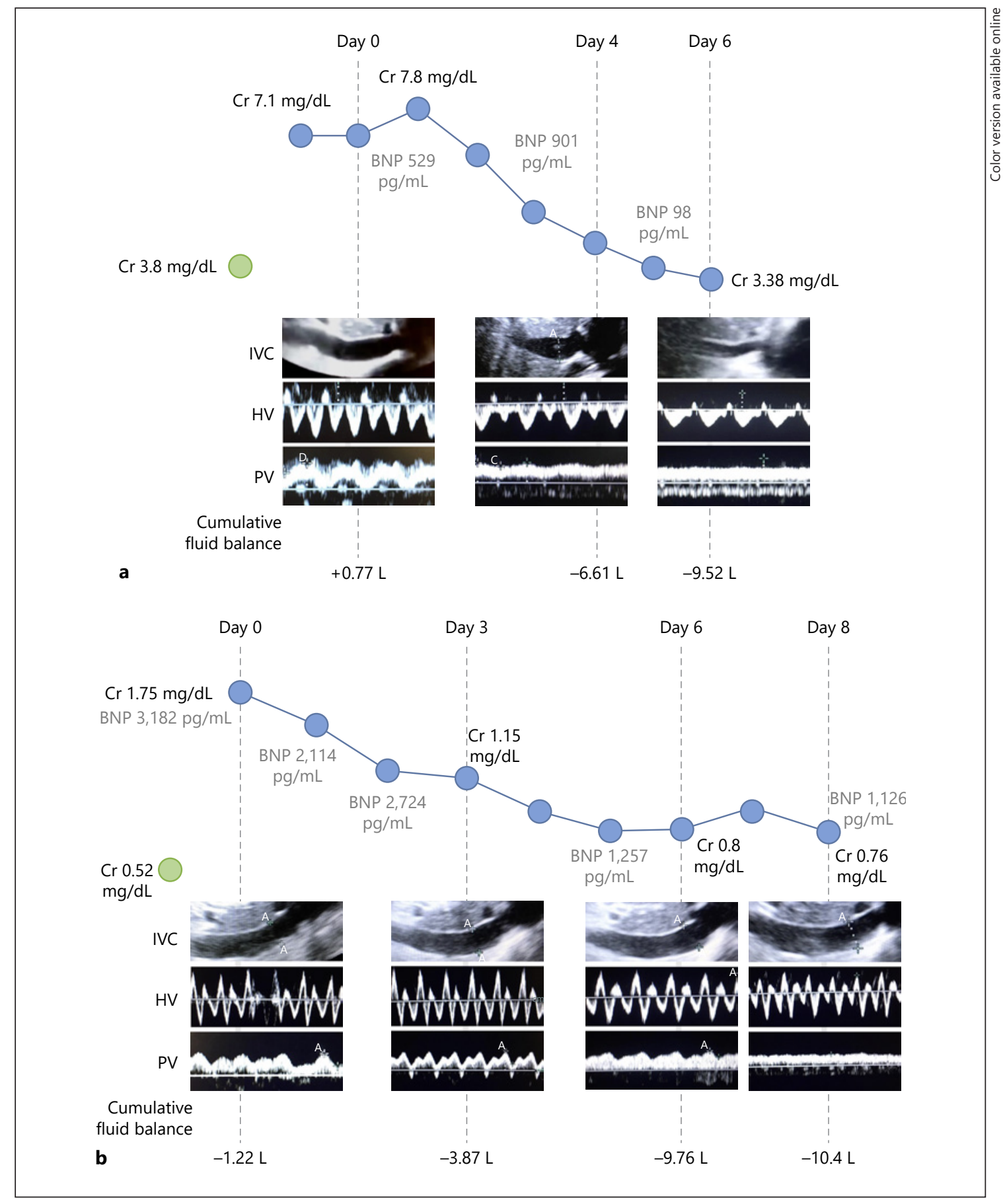

Fig. 1. a Dynamic changes in PVF pattern during decongestive treatment in a patient with HFpEF and CKD. Plethoric IVC was found at day 0 . IVC became nonplethoric after decongestion (day 6). PV PF of $46 \%$ was found on day 0 . At day 4 , PV PF improved to (20\%) and normalized by day 6 (PV PF of 9\%). Sequential changes in SCr, BNP, and cumulative fluid balance are shown. b Dynamic changes in PVF pattern during decongestive treatment in a patient with PAH. Plethoric IVC was seen at day 0 , and it did not improve with decongestion. Severe PV flow alteration was present on days 0 and 3 (PV PF 60 and 68\%, respectively) and showed progressive improvement with decongestion (32\% at day 6 and $13 \%$ at day 8 ). Sequential changes in SCr, BNP, and cumulative fluid balance are shown. Similar progressive improvement in PVF alterations was seen in 1 other patient (not shown). IVC, inferior vena cava; $\mathrm{HV}$, hepatic vein; PV, portal vein; PVF, portal vein flow, HFpEF, heart failure with preserved ejection fraction; HFrEF, heart failure with reduced ejection fraction; $\mathrm{PAH}$, pulmonary arterial hypertension; SCr, Serum Cr; PV PF, portal vein pulsatility fraction; BNP, brain natriuretic peptide.
62

Cardiorenal Med 2021;11:59-66 DOI: $10.1159 / 000511714$
Argaiz/Rola/Gamba 


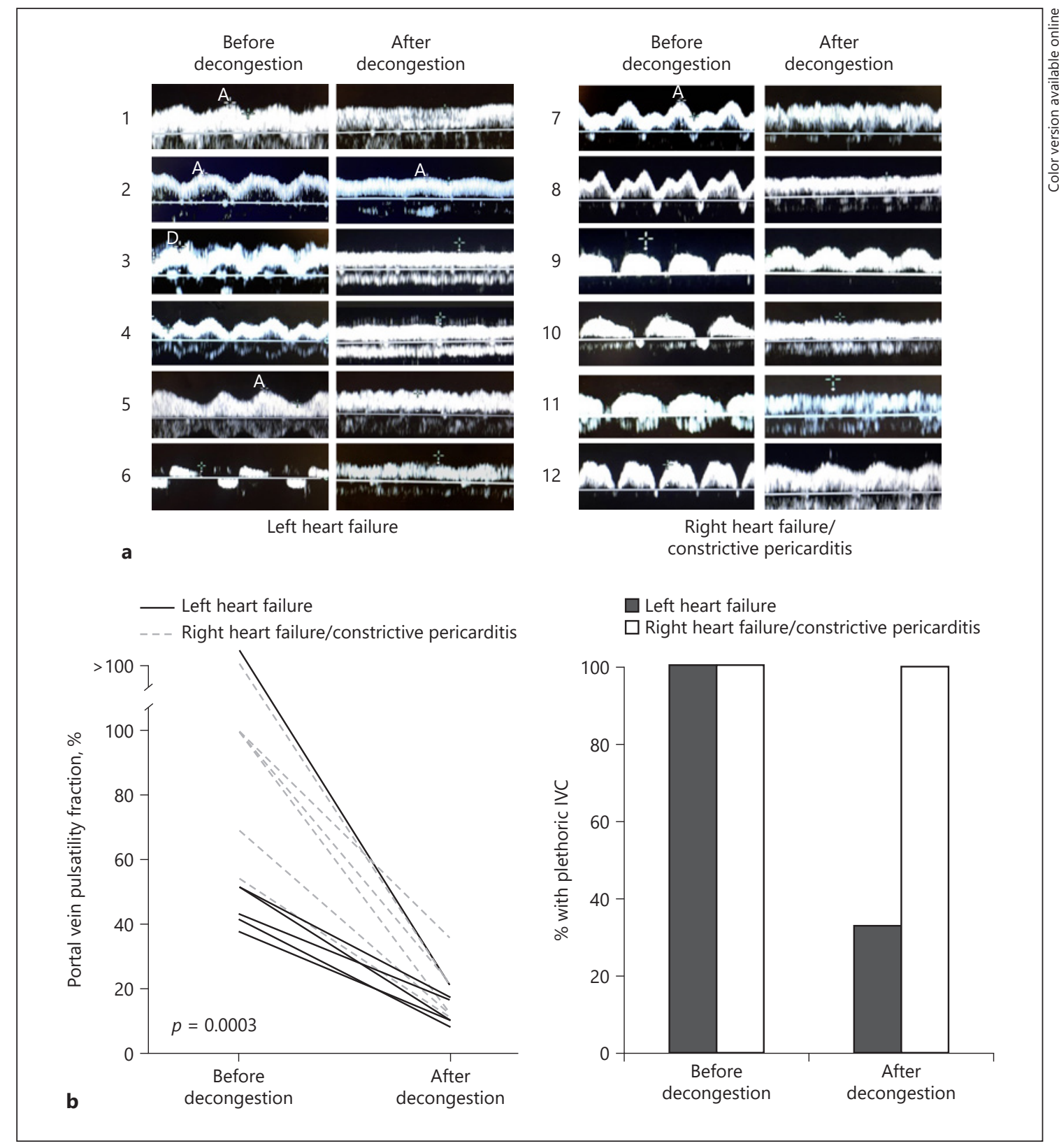

Fig. 2. a PV Doppler scans in 12 patients with venous congestion before and after decongestive treatment according to underlying cardiac pathology. b Left: quantitative change in PF ([Vmax-Vmin]Vmax $\times 100)$ with decongestive treatment. $p=0.0003$. Right: percentage of patients with plethoric IVC before and after decongestive treatment according to underlying cardiac pathology. Plethoric IVC was defined as $(>20 \mathrm{~mm}$ in diameter and $<20 \%$ inspiratory collapse). IVC, inferior vena cava; PF, pulsatility fraction.

creases the specificity of this assessment [14]. The fact that altered PVF improved considerably with diuretics in association with other signs of decongestion provides evidence that this assessment represents abdominal venous congestion in this patient population.

Portal Vein Flow Alterations during Decongestion in Congestive Heart Failure
Previous studies of abnormal venous flow patterns in heart failure have been largely associative, and currently, there is little evidence to demonstrate the reversibility of such altered patterns with any therapeutic strategy [8]. In this series, PVF alterations were completely reversed by

Cardiorenal Med 2021;11:59-66 


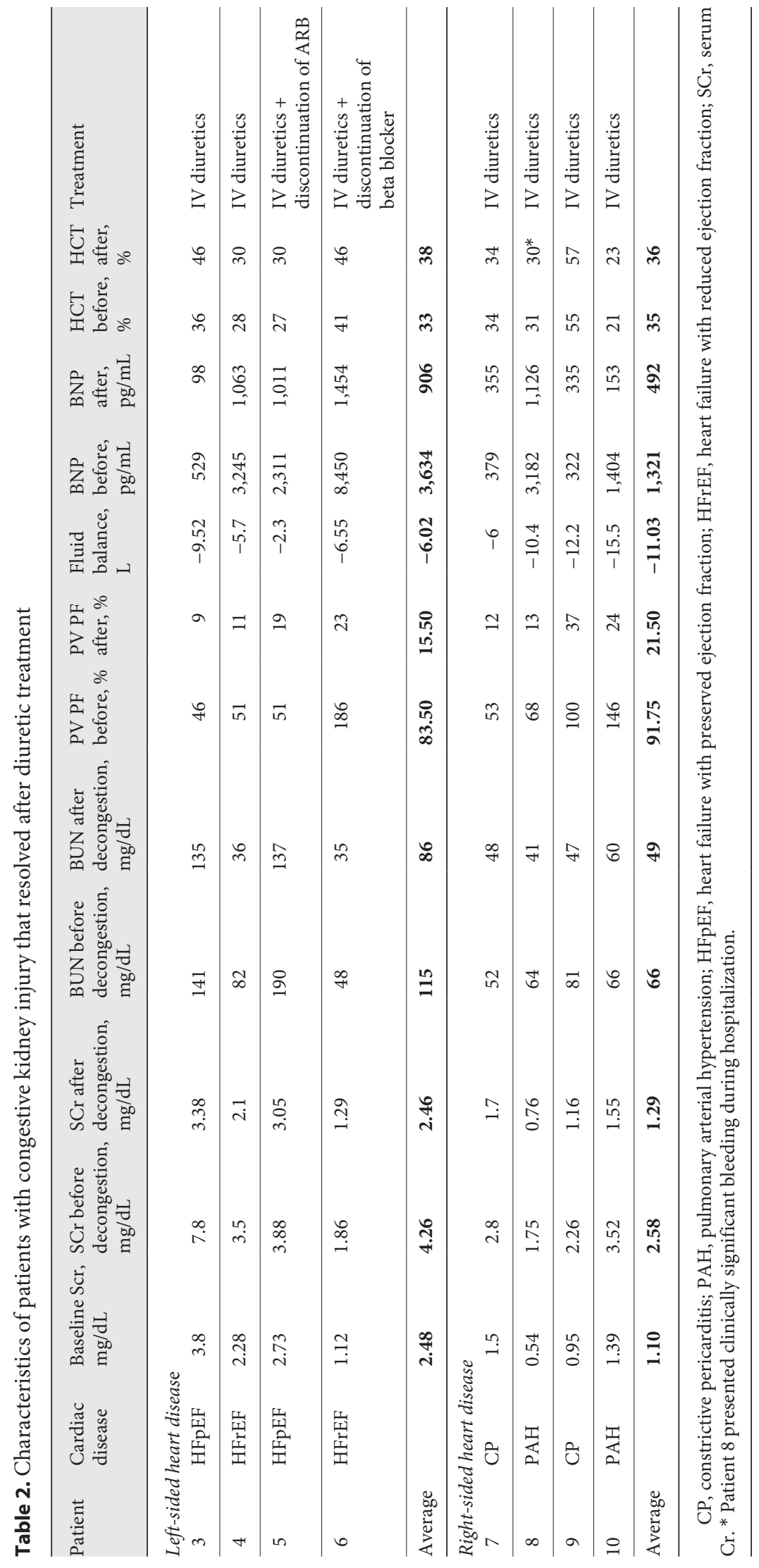


decongestion in all patients and PVF normalization coincided with return to baseline of SCr in those with congestive AKI. While there is considerable literature regarding the use of intrarenal venous Doppler in the assessment of renal-congestion $[9,10,16,17]$, PVF and IRVF alterations have been shown to correlate with each other, and PVF is easier to obtain and less time consuming than intrarenal venous Doppler, suggesting that PVF assessment might be more realistically integrated into patient care [13].

Evaluation of PV pulsatility in heart failure might add valuable information to currently established POCUS protocols like IVC and lung ultrasound (LUS). While evaluation of IVC has been proposed as a way to assess congestion in patients with heart failure $[18,19]$, the results from this series suggest that PV PF may add additional information about the degree of venous congestion that could be especially valuable when assessing patients with right-sided heart failure. LUS detection of lung congestion has become an established part of the POCUS assessment of congestion, incorporation of LUS has been shown to decrease exacerbations and urgent heart failure visits $[4,5]$. However, LUS cannot accurately distinguish cardiogenic from noncardiogenic pulmonary edema, especially in the setting of primary interstitial lung pathology [20]. Also, LUS does not evaluate abdominal venous congestion directly which is especially relevant for patients with right-sided heart failure. Given that the prevailing pathophysiology of worsening kidney injury in patients with CHF relates to increased CVP and renal venous pressure [7], assessment of PV pulsatility could represent a new bedside tool that allows the clinician to monitor abdominal congestion in real time during diagnosis and treatment.

The limitations of the present study include the lack of prospective screening of all heart failure patients presenting to the emergency department. Also, patients were only included if they had both a plethoric IVC and a PV
PF $>30 \%$. As such, the findings of this study might not apply to patients with ADHF who present with less severe congestion or who do not have both of these POCUS criteria despite severe clinical congestion.

\section{Conclusions}

Evaluation of abdominal venous congestion by POCUS could aid in diagnosis and follow-up of patients with congestive kidney injury. A plethoric IVC and an elevated PV PF in the setting of AKI should alert the clinician to the presence of venous congestion with the potential for end-organ injury. Further prospective studies are needed to evaluate the accuracy and clinical utility of this novel bedside tool.

\section{Statement of Ethics}

The Institutional Review Board approved this study with waiver of patient consent (NMM-3393).

\section{Conflict of Interest Statement}

The authors declare no conflicts of interest.

\section{Funding Sources}

The authors declare no specific funding for this study.

\section{Author Contributions}

E.R.A., P.R., and G.G. designed the study and wrote the manuscript. E.R.A. performed point-of-care ultrasound evaluations and collected pertinent patient data.

\section{References}

1 Ellison DH. Why are physicians so confused about acute heart failure? N Engl J Med. 2019; 381(24):2374-5.

2 Zile MR, Bennett TD, St John Sutton M, Cho YK, Adamson PB, Aaron MF, et al. Transition from chronic compensated to acute decompensated heart failure: pathophysiological insights obtained from continuous monitoring of intracardiac pressures. Circulation. 2008 Sep;118(14):1433-41.

3 Valle R, Aspromonte N, Milani L, Peacock FW, Maisel AS, Santini M, et al. Optimizing fluid

Portal Vein Flow Alterations during Decongestion in Congestive Heart Failure management in patients with acute decompensated heart failure (ADHF): the emerging role of combined measurement of body hydration status and brain natriuretic peptide (BNP) levels. Heart Fail Rev. 2011 Nov;16(6):519-29.

4 Rivas-Lasarte M, Álvarez-García J, Fernández-Martínez J, Maestro A, López-López L, Solé-González E, et al. Lung ultrasound-guided treatment in ambulatory patients with heart failure: a randomized controlled clinical trial (LUS-HF study). Eur J Heart Fail. 2019; 21(12):1605-13.
5 Araiza-Garaygordobil D, Gopar-Nieto R, Martinez-Amezcua P, Cabello-López A, Alanis-Estrada G, Luna-Herbert A, et al. A randomized controlled trial of lung ultrasound guided therapy in heart failure (CLUSTER-HF study). Am Heart J. 2020 Sep;227:31-9.

6 Verbrugge FH, Dupont M, Steels P, Grieten L, Malbrain M, Tang WH, et al. Abdominal contributions to cardiorenal dysfunction in congestive heart failure. J Am Coll Cardiol. 2013 Aug;62(6):485-95. 
7 Mullens W, Abrahams Z, Francis GS, Sokos G, Taylor DO, Starling RC, et al. Importance of venous congestion for worsening of renal function in advanced decompensated heart failure. J Am Coll Cardiol. 2009 Feb;53(7): 589-96.

8 Tang WH, Kitai T. Intrarenal venous flow: a window into the congestive kidney failure phenotype of heart failure? JACC Heart Fail. 2016;4(8):683-6.

9 Iida N, Seo Y, Sai S, Machino-Ohtsuka T, Yamamoto $\mathrm{M}$, Ishizu $\mathrm{T}$, et al. Clinical implications of intrarenal hemodynamic evaluation by Doppler ultrasonography in heart failure. JACC Heart Fail. 2016;4(8):674-82.

10 Husain-Syed F, Birk HW, Ronco C, Schörmann T, Tello K, Richter MJ, et al. Dopplerderived renal venous stasis index in the prognosis of right heart failure. J Am Heart Assoc. 2019 Nov;8(21):e013584.

11 Eljaiek R, Cavayas YA, Rodrigue E, Desjardins G, Lamarche Y, Toupin F, et al. High postoperative portal venous flow pulsatility indicates right ventricular dysfunction and predicts complications in cardiac surgery patients. Br J Anaesth. 2019 Feb;122(2):206-14.
12 Singh NG, Kumar KN, Nagaraja PS, Manjunatha N. Portal venous pulsatility fraction, a novel transesophageal echocardiographic marker for right ventricular dysfunction in cardiac surgical patients. Ann Card Anaesth. 2020 Mar;23(1):39-42.

13 Beaubien-Souligny W, Benkreira A, Robillard P, Bouabdallaoui N, Chassé M, Desjardins G, et al. Alterations in portal vein flow and intrarenal venous flow are associated with acute kidney injury after cardiac surgery: a prospective observational cohort study. J Am Heart Assoc. 2018 Oct; 7(19):e009961.

14 Beaubien-Souligny W, Rola P, Haycock K, Bouchard J, Lamarche Y, Spiegel R, et al. Quantifying systemic congestion with pointof-care ultrasound: development of the venous excess ultrasound grading system. Ultrasound J. 2020;12(1):16.

15 Gallix BP, Taourel P, Dauzat M, Bruel JM, Lafortune M. Flow pulsatility in the portal venous system: a study of Doppler sonography in healthy adults. AJR Am J Roentgenol. 1997 Jul;169(1):141-4.

16 Puzzovivo A, Monitillo F, Guida P, Leone M, Rizzo C, Grande D, et al. Renal venous pat- tern: a new parameter for predicting prognosis in heart failure outpatients. J Cardiovasc Dev Dis. 2018 Nov;5(4):52.

17 Nijst P, Martens P, Verbrugge F, Dupont M, Tang WH, Mullens W. Intravascular volume expansion in patients with heart failure and reduced ejection fraction is not revealed by changes in cardiac filling pressures. J Am Coll Cardiol. 2019 Jun;69(11):735.

18 Carbone F, Bovio M, Rosa GM, Ferrando F, Scarrone A, Murialdo G, et al. Inferior vena cava parameters predict re-admission in ischaemic heart failure. Eur J Clin Invest. 2014 Apr;44(4):341-9.

19 Goonewardena SN, Gemignani A, Ronan A, Vasaiwala S, Blair J, Brennan JM, et al. Comparison of hand-carried ultrasound assessment of the inferior vena cava and $\mathrm{N}$-terminal pro-brain natriuretic peptide for predicting readmission after hospitalization for acute decompensated heart failure. JACC Cardiovasc Imaging. 2008 Sep;1(5):595-601.

20 Koratala A, Ronco C, Kazory A. Need for objective assessment of volume status in critically ill patients with COVID-19: the tri-POCUS approach. Cardiorenal Med. 2020;10(4):209-16. 